\title{
ESTILO DE VIDA DE GESTANTES ATENDIDAS NA ATENÇÃO PRIMÁRIA À SAÚDE DE UMA CAPITAL BRASILEIRA
}

\section{Lifestyle of pregnant women served by the primary health care of a Brazilian capital \\ Estilo de vida de embarazadas de la atención primaria de salud de una capital brasileña}

Luilma Albuquerque Gurgel

Universidade Estadual do Ceará - UECE - Fortaleza (CE) - Brasil

Levi de Holanda Francalino

Universidade Estadual do Ceará - UECE - Fortaleza (CE) - Brasil

Jaina Bezerra Aguiar

Universidade Estadual do Ceará - UECE - Fortaleza (CE) - Brasil

Janalu Bernardo Guimarães

Universidade Estadual do Ceará - UECE - Fortaleza (CE) - Brasil

\section{Luana Maria Lopes Farias}

Universidade Estadual do Ceará - UECE - Fortaleza (CE) - Brasil

Regikyslla Erika Ferreira Rodrigues

Universidade Estadual do Ceará - UECE - Fortaleza (CE) - Brasil

Ana Luisa Batista Santos

Universidade Estadual do Ceará - UECE - Fortaleza (CE) - Brasil

\section{RESUMO}

Objetivo: Avaliar o estilo de vida de usuárias gestantes atendidas no âmbito da atenção primária à saúde da rede pública. Métodos: Tratase de estudo de natureza descritiva, abordagem quantitativa e corte transversal, realizado em 12 Unidades Básicas de Saúde de Fortaleza (Ceará), no período de dezembro de 2015 a abril de 2016, com 107 gestantes. Utilizou-se o formulário Estilo de Vida Fantástico, que possui 25 itens divididos em nove domínios. As respostas de cada item possuem pontuação que varia de 0 a 4 pontos. A soma de todos os pontos permite classificar o estilo de vida em Excelente ( 85 a 100 pontos), Muito bom (70 a 84 pontos), Bom (55 a 69 pontos), Regular (35 a 54 pontos) e Necessita melhorar ( 0 a 34 pontos). Aplicou-se análise estatística descritiva dos dados quantitativos para apresentação de resultados utilizando medidas de tendência central (média) e medidas de dispersão (porcentagem, desvio padrão e valor máximo). Resultados: A média geral obtida $(72,26$ pontos) enquadrou o grupo na categoria Muito bom. $9 \%(n=10)$ das gestantes encontraram-se na categoria Excelente e $6 \%(\mathrm{n}=6)$ na categoria Regular. Nenhuma gestante teve seu estilo de vida classificado como Necessita melhorar. Atividade física apresentou a menor média, enquanto o álcool obteve a média mais elevada, havendo grande disparidade entre elas. Conclusão: Apesar de o estilo de vida ter sido classificado como adequado, aspectos relevantes para a saúde materno-infantil, como atividade física e nutrição apresentaram pontuações abaixo das esperadas.

Descritores: Gravidez; Estilo de Vida; Cuidado Pré-Natal.

\section{ABSTRACT}

Objective: To evaluate the lifestyle of pregnant women cared for within the public primary health care network. Methods: This is a descriptive study with quantitative and cross-sectional approach, carried out in 12 Basic Health Units of Fortaleza (Ceará), in the period from December 2015 to April 2016, with 107 pregnant women. It used the Fantastic Lifestyle form, which comprises 25 items divided into nine domains. The answers to each item have scores ranging from 0 to 4 points. The sum of all points allows lifestyle to be rated into Excellent (85 to 100 points), Very good (70 to 84 points), Good (55 to 69 points), Regular (35 to 54 points,) and Needs improvement (0 to 34 points). Descriptive statistical analysis was applied in order to present the results through measures of central tendency (mean) and measures of spread (percentage, standard deviation, and maximum value). Results: The overall average (72.26 points) ranked the group under the category Very good. Of the pregnant women, $9 \%(n=10)$ were found in the category Excellent and $6 \%(n=6)$ in the category Regular. No pregnant woman had her lifestyle classified as Needs improvement. Physical activity presented the lowest mean, while alcohol had the highest mean, with great 
disparity between them. Conclusion: Although the lifestyle was classified as adequate, relevant domains to maternal and child health, such as physical activity and nutrition, showed below-expected scores.

Descriptors: Pregnancy; Life Style; Prenatal Care.

\section{RESUMEN}

Objetivo: Evaluar el estilo de vida de las usuarias embarazadas que son asistidas en la atención primaria de salud de la red pública. Métodos: Se trata de un estudio descriptivo, de abordaje cuantitativo y corte transversal realizado en 12 Unidades Básicas de Salud de Fortaleza, Ceará en el período entre diciembre de 2015 y abril de 2016 con 107 embarazadas. Se utilizó el formulario Estilo de Vida Fantástico que tiene 25 items divididos en nueve dominios. Las respuestas de cada item tiene la puntuación entre 0 y 4 puntos. La suma de todos los puntos permite la clasificación del estilo de vida en Excelente (entre 85 y 100 puntos), Muy bueno (entre 70 y 84 puntos), Bueno (entre 55 y 69 puntos), Regular (entre 35 y 54 puntos) y Necesita mejorías (entre 0 y 34 puntos). Se realizó un análisis descriptivo de los datos cuantitativos para la presentación de los resultados utilizándose las medidas de tendencia central (media) y medidas de dispersión (el porcentaje, la desviación típica y el valor máximo). Resultados: La media global (72,26 puntos) ha puesto el grupo en la categoría Muy bueno. El 9\% $(n=10)$ de las embarazadas estaban en la categoría Excelente y el $6 \%(n=6)$ en la Regular. Ninguna embarazada tuvo su estilo de vida clasificado en Necesita mejoría. La actividad física presentó la media más baja mientras el alcohol tuvo la media más elevada con gran diferencia entre ellos. Conclusión: Aunque el estilo de vida haya sido clasificado como adecuado los aspectos relevantes para la salud materno-infantil como la actividad física y la nutrición presentaron puntuaciones abajo de las que se esperaba.

Descriptores: Embarazo; Estilo de Vida; Atención Prenatal.

\section{INTRODUÇÃO}

A promoção da saúde mostra-se como uma das ideias centrais do discurso da saúde pública das últimas décadas, numa perspectiva de redirecionamento de suas práticas ${ }^{(1)}$. Destacam-se alterações nas concepções relacionadas à saúde maternoinfantil, especificamente com relação ao papel do estilo de vida adotado pela mãe na determinação da saúde e da doença. Sendo a gravidez um período intenso de mudanças, de descobertas e de aprendizados, torna-se também uma oportunidade para o investimento em estratégias de educação e de cuidado em saúde ${ }^{(2)}$.

Inicialmente, toda gravidez era considerada um risco devido à possibilidade de ocorrência de dano biológico para ambos: mãe e filho. Pouca preocupação era dada aos fatores socioeconômicos, mas isso mudou a partir da década de 70, e mulheres com baixa renda passaram a ser a população alvo para esses riscos. Recentemente, o critério utilizado para definir os riscos não é mais a renda familiar, já que sozinhos os fatores econômicos não podem predizer o resultado de saúde e de doença em determinado grupo social. O foco atual é a junção das influências dos fatores psicológicos, das condições socioeconômicas e do modelo de $\operatorname{vida}^{(3)}$. Nessa perspectiva, mais abrangente, faz-se referência às condições gerais de vida que incluem vários elementos: renda familiar, emprego, alimentação, moradia, condições sanitárias, acesso aos serviços de saúde e educação materna ${ }^{(4)}$.

O estilo de vida é caracterizado por padrões de comportamento que podem ser identificados, podendo ter um forte efeito na saúde, e estarem relacionados a vários aspectos que refletem as atitudes, os valores e as oportunidades na vida das pessoas ${ }^{(5,6)}$. Hábitos alimentares e de atividade física, por exemplo, são elementos do estilo de vida que desempenham uma função importante na promoção da saúde e na prevenção de doenças ${ }^{(7)}$. Além desses, outros elementos do estilo de vida são importantes para a saúde e para o bem-estar: evitar o uso de cigarros e o consumo de álcool, possuir relacionamento harmonioso com a família e com os amigos, praticar sexo seguro e gerenciar o estresse, além da necessidade de se ter uma visão otimista e positiva da $\operatorname{vida}^{(8)}$.

O nascimento de uma criança marca a vida da mulher como sendo um período pautado por muitas mudanças e com enorme impacto na rotina pessoal e familiar. A mulher grávida, além de ter de se adaptar às mudanças físicas provocadas pelo parto, depara-se com um novo ser que depende dela. Portanto, sendo a gravidez uma época de profundas alterações físicas e psicológicas na vida da mulher, pode e deve ser também uma oportunidade para a adoção de estilo de vida mais saudável ${ }^{(9)}$.

As ações mais importantes para o êxito no atendimento das gestantes e no estímulo ao estilo de vida mais saudável são dependentes do acesso e da qualidade da atenção realizada pelos serviços de saúde, especialmente na atenção ao pré-natal, ao parto e ao puerpério. O acompanhamento ao pré-natal tem impacto na redução da mortalidade materna e perinatal, desde que as mulheres tenham acesso aos serviços, os quais devem ter qualidade suficiente para o controle dos riscos identificados, sendo, portanto, fundamental para a promoção da saúde materno-infantil ${ }^{(10)}$.

Nesse sentido, a atuação dos profissionais que desenvolvem as consultas de pré-natal deve considerar orientações em todos os domínios do estilo de vida, mas, para isso, é necessário identificar o comportamento das gestantes em cada um desses domínios, o que justifica a realização do presente estudo. Desta feita, será possível propor intervenções mais direcionadas 
às necessidades das gestantes e que sejam capazes de promover saúde para mães e filhos, contribuindo com o estilo de vida das gestantes. Além disso, o resultado da presente pesquisa norteará o trabalho da referida equipe, contribuindo assim com a construção de uma rede de saúde de melhor qualidade, mais humanizada e engajada na compreensão do ser de modo integral.

À vista do exposto, o presente trabalho objetiva avaliar o estilo de vida de usuárias gestantes atendidas no âmbito da atenção primária à saúde da rede pública.

\section{MÉTODOS}

Trata-se de estudo de caráter descritivo, com abordagem quantitativa e de corte transversal. Realizou-se a coleta de dados em todas as 12 Unidades Básicas de Saúde (UBS) da Regional administrativa IV de Fortaleza, Ceará. O município possui 86 UBS, distribuídas em 6 Regionais, sendo a selecionada para a coleta de dados a que abriga a instituição de ensino superior responsável pela pesquisa.

A coleta dos dados ocorreu entre os meses de dezembro de 2015 e de abril de 2016. Definiram-se previamente os dias e os horários determinados para a coleta de dados, de forma a coincidirem com os turnos de realização das consultas de pré-natal nas UBS.

A amostra formou-se por gestantes que já tinham realizado pelo menos uma consulta pré-natal, nulíparas, ou multíparas, com qualquer nível de escolaridade, cuja gestação estivesse no mínimo no quarto mês e com idade entre 19 e 35 anos. Como critérios de exclusão do estudo, consideraram-se gestantes com gravidez classificada pelo médico como sendo de risco (dado obtido com a própria gestante), que possuíam problemas de fala, de audição e/ou intelectuais que pudessem impossibilitar a aplicação do formulário, bem como aquelas com alterações motoras que pudessem comprometer a realização de atividades físicas, como hemiplegia, paraplegia, fratura em consolidação de membro inferior, dentre outras.

Buscou-se a relação das gestantes atendidas em cada unidade básica. Dessa forma, realizou-se o cálculo amostral para população infinita, obtendo-se o valor 107. Consideraram-se o nível de confiança de $95 \%$ e o erro máximo desejado de 0,05 , com desvio padrão de 0,26 .

Para a captação de dados referentes ao estilo de vida das gestantes, utilizou-se o formulário Estilo de Vida Fantástico ${ }^{(11)}$, traduzido para o português $\mathrm{s}^{(12)}$. Este é um instrumento abrangente, desenvolvido pelo Departamento de Medicina Familiar da Universidade McMaster (Canadá), que tem como finalidade auxiliar médicos que trabalham com a prevenção para que possam avaliar o estilo de vida dos pacientes e os possa conhecer melhor ${ }^{(11)}$.

O instrumento possui 25 itens divididos em nove domínios: 1) família e amigos; 2) atividade física; 3) nutrição; 4) cigarro e drogas; 5) álcool; 6) sono, cinto de segurança, estresse e sexo seguro; 7) tipo de comportamento; 8) introspecção e 9) trabalho. A pontuação de cada item varia de 0 a 4 pontos. A análise dos itens separadamente possibilita um olhar mais aprofundado para os diversos comportamentos das gestantes relacionados a cada um dos domínios do estilo de vida.

Os domínios deram origem à palavra fantástico, originada do acrônimo fantastic, que representa as letras iniciais dos nomes dos nove domínios na língua inglesa: $\mathrm{F}=$ Family and friends (família e amigos); $\mathrm{A}=$ Activity (atividade física); $\mathrm{N}=$ Nutrition (nutrição); $\mathrm{T}=$ Tobacco \& toxics (cigarro e drogas); $\mathrm{A}=$ Alcohol (álcool); $\mathrm{S}=$ Sleep, seatbelts, stress, safe sex (sono, cinto de segurança, estresse e sexo seguro); $\mathrm{T}$ = Type of behavior (tipo de comportamento; padrão de comportamento A ou B); $\mathrm{I}=$ Insight (introspecção) e C = Career (trabalho; satisfação com a profissão).

O instrumento considera o comportamento das gestantes e os seus resultados permitem determinar a associação entre o estilo de vida e a saúde. Os itens estão dispostos na forma de escala Likert, onde 23 possuem cinco alternativas de resposta e 2 são dicotômicos. As alternativas estão dispostas na forma de colunas para facilitar a sua codificação e a alternativa da esquerda é sempre a de menor valor, ou de menor relação, com um estilo de vida saudável.

A codificação dos itens é realizada por pontos, da seguinte maneira: 0 para a primeira coluna, 1 para a segunda coluna, 2 para a terceira coluna, 3 para a quarta coluna e 4 para a quinta coluna. Os itens que só possuem duas alternativas pontuam: zero para a primeira coluna e 4 pontos para a última coluna. A soma de todos os pontos permite chegar a um escore total que classifica os indivíduos em cinco categorias, quais sejam: Excelente ( 85 a 100 pontos), Muito bom (70 a 84 pontos), Bom (55 a 69 pontos), Regular ( 35 a 54 pontos) e Necessita melhorar ( 0 a 34 pontos).

Para a análise dos dados calcularam-se os escores de cada domínio do estilo de vida presentes no formulário de cada gestante, bem como o somatório geral dos mesmos. Tabularam-se os dados obtidos para meio eletrônico na forma de planilhas posteriormente migrados para o programa estatístico Statistical Package for the Social Sciences (SPSS for Windows, versão 17.0). Aplicou-se análise estatística descritiva dos dados quantitativos para apresentação de resultados utilizando medidas de tendência central (média) e medidas de dispersão (porcentagem, desvio padrão e valor máximo).

A pesquisa está em conformidade com a Resolução n 466/12 do Conselho Nacional de Saúde, tendo sido aprovada pelo Comitê de Ética da Universidade Estadual do Ceará sob o Parecer nº 1.430.328. 


\section{RESULTADOS}

Participaram do estudo 107 gestantes, das quais 42,1\% ( $\mathrm{n}=45)$ possuíam idade entre 20 e 24 anos, 42,1\% ( $\mathrm{n}=45)$ encontravam-se no $4^{\circ}$ ou $5^{\circ}$ mês da gestação e $34,6 \%(n=37)$ vivenciavam a $2^{a}$ gestação. Ainda $57,1 \%(n=61)$ das gestantes concluíram o ensino médio e 74,8\% (n=80) possuíam renda familiar de 1 a 2 salários mínimos (Tabela I).

Tabela I - Caracterização da amostra segundo a idade, mês da gestação, número de gestações anteriores, escolaridade e renda familiar. Fortaleza, Ceará, 2016.

\begin{tabular}{lcc}
\hline Caracterização da amostra & $\mathbf{n}$ & $\mathbf{\%}$ \\
\hline Idade (anos completos) & & \\
$20-24$ & 45 & 42,1 \\
$25-29$ & 38 & 35,5 \\
$30-35$ & 24 & 22,4 \\
Mês da gestação & 45 & 42,1 \\
$4^{\circ}-5^{\circ}$ & 30 & 28,0 \\
$6^{\circ}-7^{\circ}$ & 32 & 29,9 \\
$8^{\circ}-9^{\circ}$ & & \\
Gestaçóes anteriores & 24 & 22,4 \\
Nenhuma & 37 & 34,6 \\
Uma & 31 & 29,0 \\
Duas & 11 & 10,3 \\
Três & 4 & 3,7 \\
Quatro & & \\
Escolaridade & 10 & 9,3 \\
Ensino fundamental incompleto & 11 & 10,3 \\
Ensino fundamental completo & 15 & 14,0 \\
Ensino médio incompleto & 61 & 57,1 \\
Ensino médio completo & 2 & 1,9 \\
Superior incompleto & 8 & 7,4 \\
Superior completo & & 2,8 \\
Renda familiar & 3 & 10,3 \\
Nenhuma & 11 & 74,8 \\
Menos de um salário mínimo & 80 & 11,2 \\
1 a 2 salários mínimos & 12 & 0,9 \\
3 a 4 salários mínimos & 1 & \\
5 a 6 salários mínimos & & \\
\hline
\end{tabular}

Em relação à classificação individual das gestantes, $59 \%(\mathrm{n}=63)$ apresentaram o estilo de vida classificado como Muito bom e $26 \%(n=28)$ encontravam-se na categoria Bom. Ainda $9 \%(n=10)$ e $6 \%(n=6)$ apresentaram, respectivamente, estilo de vida Excelente e Regular.

Cada domínio do estilo de vida possui uma pontuação máxima própria que varia de acordo com a quantidade de itens existentes. Para cada um calculou-se a média obtida, sendo essa representada na forma de percentual em relação à nota máxima possível para o domínio (Tabela II).

Tabela II - Domínio, número de itens, nota máxima possível, média obtida e percentual máximo obtido do estilo de vida de gestantes atendidas nos postos de saúde. Fortaleza, Ceará, 2016.

\begin{tabular}{lcccc}
\hline Domínio & $\begin{array}{c}\mathbf{N}^{\circ} \mathbf{d e} \\
\text { Itens }\end{array}$ & $\begin{array}{c}\text { Nota } \\
\text { máxima }\end{array}$ & $\begin{array}{c}\text { Média } \\
\text { obtida }\end{array}$ & $\begin{array}{c}\text { Pmáx } \\
\text { (\%) }\end{array}$ \\
\hline Família e amigos & 2 & 8 & 6,77 & 84,62 \\
Atividade física & 2 & 8 & 3,20 & 40 \\
Nutrição & 3 & 12 & 7,62 & 63,58 \\
Cigarro e drogas & 4 & 16 & 14,13 & 88,31 \\
Álcool & 3 & 12 & 11,81 & 98,41 \\
Sono, cinto de segurança, estresse e sexo seguro & 5 & 20 & 12,70 & 63,20 \\
Tipo de comportamento & 2 & 8 & 4,71 & 58,87 \\
Introspecção & 3 & 12 & 8,41 & 70,08 \\
Trabalho & 1 & 4 & 2,91 & 72,75 \\
Total & 25 & 100 & 72,26 & 72,26 \\
\hline
\end{tabular}

Pmáx = Média obtida representada em percentual em relação à nota máxima possível para o domínio. 
Igualmente, calculou-se a pontuação média de cada um dos 25 itens do formulário (Tabela III). A amostra obteve 2,89 pontos na média geral de pontos por item do formulário.

Tabela III - Pontuação média e desvio padrão para cada item do estilo de vida de gestantes atendidas nos postos de saúde. Fortaleza, Ceará, 2016.

\begin{tabular}{|c|c|c|}
\hline Item & Pontuação média & Desvio padrão \\
\hline \multicolumn{3}{|l|}{ Família e amigos } \\
\hline Tenho alguém para conversar & 3,26 & 1,20 \\
\hline Dou e recebo afeto & 3,50 & 0,93 \\
\hline \multicolumn{3}{|l|}{ Atividade física } \\
\hline Sou vigorosamente ativa & 0,30 & 0,83 \\
\hline Sou moderadamente ativa & 2,90 & 1,45 \\
\hline \multicolumn{3}{|l|}{ Nutrição } \\
\hline Como uma dieta balanceada & 2,31 & 1,47 \\
\hline Excesso de açúcar, sal, gordura & 3,00 & 1,21 \\
\hline Peso corporal saudável & 2,32 & 1,55 \\
\hline \multicolumn{3}{|l|}{ Cigarro e drogas } \\
\hline Fumo cigarro & 3,69 & 0,84 \\
\hline Uso drogas & 3,74 & 1,00 \\
\hline Abuso de remédios & 3,81 & 0,73 \\
\hline Bebo café, chá e colas & 2,89 & 0,68 \\
\hline \multicolumn{3}{|l|}{ Álcool } \\
\hline Consumo por semana (álcool) & 3,96 & 0,27 \\
\hline Consumo por ocasião (álcool) & 3,85 & 0,58 \\
\hline Dirijo após beber & 4,00 & 0,00 \\
\hline \multicolumn{3}{|c|}{ Sono, cinto de segurança, estresse e sexo seguro } \\
\hline Durmo bem & 2,30 & 1,54 \\
\hline Uso cinto de segurança & 3,18 & 1,42 \\
\hline Sou capaz de lidar com meu estresse & 2,63 & 1,32 \\
\hline Relaxo no meu tempo de lazer & 2,71 & 1,39 \\
\hline Pratico sexo seguro & 1,89 & 1,72 \\
\hline \multicolumn{3}{|l|}{ Tipo de comportamento } \\
\hline Aparento estar com pressa & 2,44 & 1,63 \\
\hline Sinto-me com raiva e hostil & 2,27 & 1,38 \\
\hline \multicolumn{3}{|l|}{ Introspecção } \\
\hline Penso de forma positiva & 3,21 & 1,11 \\
\hline Sinto-me tensa e desapontada & 2,50 & 1,36 \\
\hline Sinto-me triste e deprimida & 2,70 & 1,37 \\
\hline \multicolumn{3}{|l|}{ Trabalho } \\
\hline Estou satisfeita com meu trabalho & 2,91 & 1,60 \\
\hline
\end{tabular}

$\mathrm{Na}$ categoria Introspecção, encontraram-se particularidades em relação às suas respostas. Os itens "Sinto-me tensa e desapontada" e "Sinto-me triste e deprimida" apresentam prevalência de 17,8\% (n=19) e 14\% (n=15) respectivamente, em respostas de notas 0 (quase sempre) e 1 (com relativa frequência), aumentando para $48,6 \%$ ( $n=52)$ e $44,9 \%$ ( $=48$ ) considerando também respostas com nota 2 (algumas vezes).

\section{DISCUSSÃO}

Identificou-se na análise dos dados que domínios do estilo de vida das gestantes avaliadas na presente pesquisa (Atividade física; Nutrição; Sono, cinto de segurança, estresse e sexo seguro e Tipo de comportamento) necessitam ser trabalhados durante as consultas de pré-natal de modo a melhorar o estilo de vida delas, promovendo saúde para o binômio mãe-filho.

No domínio Família e amigos do presente estudo, quando se avaliou a frequência de troca de afeto e de conversas intimistas, o Pmáx obtido corresponde a $84,62 \%$, o que indica uma conduta com adequada influência para a saúde.

O domínio Atividade física obteve média geral de 3,2 (Pmáx $=40 \%)$, sendo inferior aos 5,11 encontrados em indivíduos adultos saudáveis ${ }^{(14)}$ e aos 4,32 obtidos por estudantes universitários ${ }^{(13)}$. Tal valor é próximo ao de 2,8 encontrado em estudantes de medicina do $5^{\circ}$ ao $8^{\circ}$ período $^{(14)}$.

Estudos conduzidos em populações gestantes encontraram baixo nível de atividade física ${ }^{(15,16)}$. Identificou-se que o padrão de atividade física de gestantes do município de Campina Grande (Paraíba) encontrava-se baixo desde o primeiro trimestre gestacional, diminuindo ainda mais ao passar do tempo, até chegar a $100 \%$ da amostra em padrão sedentário a partir da $32^{\text {a }}$ 
semana $^{(15)}$. Uma pesquisa realizada também na Regional IV de Fortaleza obteve 80,3\% da amostra em níveis de atividade física considerados sedentário ou leve, demonstrando prevalência de inatividade no período gestacional, apresentando correlação entre atividade física e trimestre gestacional ${ }^{(16)}$.

No formulário Estilo de Vida Fantástico, o domínio Atividade física possui 2 itens. Dentre estes, o item "Sou vigorosamente ativa" obteve a menor média (0,3 pontos). Em contrapartida o item "Sou moderadamente ativa" obteve média maior (2,9 pontos), o qual considera o trabalho doméstico como atividade moderada ${ }^{(11)}$.

O domínio Nutrição, do formulário Estilo de Vida Fantástico, questiona a frequência de consumo de uma dieta balanceada, o excesso de açúcar, de sal, de gordura animal, de bobagens e de salgadinhos, bem como o intervalo de peso em que o indivíduo se encontra em relação ao ideal. O percentual encontrado na atual pesquisa nesse domínio (Pmáx = 63,58\%) figurou abaixo da média percentual total do instrumento (72,26\%). Mesmo que o estado nutricional e o ganho de peso nesse período sejam cruciais, tanto para a mãe quanto para o feto, ainda não há comportamento satisfatoriamente adequado em relação a esse item ${ }^{(17)}$.

Os domínios Cigarro e drogas e Álcool apresentaram as maiores porcentagens (Pmáx = 88,31\% e 98,41\%, respectivamente) no estudo em questão. Em estudo realizado na Maternidade Escola da Universidade Federal do Rio de Janeiro (UFRJ), 5,5 e $7,4 \%$ das puérperas relataram, respectivamente, o uso de cigarro e de álcool durante a gestação. Ainda nesse estudo, os autores verificaram que a assistência nutricional teve um efeito protetor contra o tabagismo, concluindo que as gestantes devem ser esclarecidas quanto aos possíveis riscos dessas substâncias ${ }^{(18)}$. Pode-se perceber na presente pesquisa o cuidado das gestantes em relação ao consumo desses itens. Destaca-se ainda que o item referente ao consumo de café, de chás e de colas obteve a menor pontuação nesses dois domínios $(2,89)$.

O domínio Sono, cinto de segurança, estresse e sexo seguro obteve porcentagem no presente estudo de $63,2 \%$. Uma pesquisa realizada com gestantes residentes de Alfenas (Minas Gerais), no segundo ou no terceiro trimestre de gestação, relatou como principais motivos da diminuição da qualidade do sono o aumento da frequência urinária noturna e a preocupação com a saúde e com o nascimento do bebê $\hat{e}^{(19)}$. Há evidências que o estresse durante a gestação e durante os primeiros anos de vida pode ser um fator relacionado ao desenvolvimento do câncer ${ }^{(20)}$.

O item Pratico sexo seguro apresentou o segundo menor escore $(1,89)$ na presente pesquisa, evidenciando a necessidade de empoderamento das gestantes de Fortaleza acerca dos riscos ao feto advindos da prática de sexo sem segurança durante a gestação. Uma possível explicação para esse resultado pode ser o fato de que as mulheres vêem os métodos de prevenção apenas como contraceptivos, sem se dar conta dos riscos associados à prática sexual sem segurança, riscos estes há muito tempo conhecidos. As gestantes e suas parcerias sexuais devem ser investigadas e informadas sobre as infecções sexualmente transmissíveis, tendo em vista que a presença na gravidez pode causar aborto, parto prematuro, ou doenças congênitas, bem como ter efeitos debilitantes na gestante ${ }^{(21)}$.

A média de escores do domínio Tipo de comportamento apresentou o segundo menor percentual em relação à nota máxima possível $(58,87 \%)$, tendo obtido média de escore de 4,71 . Encontrou-se resultados aproximados em grupo de hipertensos $(4,69)$ (13) e em grupo de estudantes de medicina $(4,2)^{(14)}$.

Na presente pesquisa, o domínio Introspecção apresentou um percentual abaixo da média geral (70,08\%). Apesar desse valor, para os dois itens que avaliam a sensação de tristeza, de desapontamento e de depressão, 48,6\% (n=52) e 44,9\% (n=48), respectivamente, das gestantes responderam com as opções Quase sempre, Com relativa frequência ou Algumas vezes.

Estudos têm se voltado para a depressão no período gestacional na atenção básica à saúde ${ }^{(22-24)}$. Tais pesquisadores identificaram que fatores sociodemográficos, como o nível de pobreza, a história psiquiátrica, a ausência do parceiro e os eventos vitais estressantes, têm relação com a depressão pós-parto(22). Além disso, verificaram que mulheres com depressão pré-natal apresentaram 2,4 vezes mais chances de apresentar sintomas depressivos pós-parto ${ }^{(23)}$. Há relação entre a depressão na gravidez e o baixo peso ao nascer, além da prematuridade ${ }^{(24)}$.

Os escores do domínio Trabalho atingiram o percentual de $72,75 \%$ da pontuação máxima possível no presente trabalho. Esse domínio, no formulário Estilo de Vida Fantástico, possui apenas um item e, por isso, pode não refletir de maneira integral a relação das gestantes com o seu trabalho.

Em estudo realizado com gestantes atendidas em Unidades Básicas de Saúde no município de Itabuna (Bahia), onde as atividades domésticas eram realizadas por $62,9 \%$ das gestantes, encontrou-se uma prevalência altamente significativa entre a dor lombar e a atividade doméstica ${ }^{(25)}$. Outros estudos devem ser realizados buscando entender a falta de adesão aos exercícios físicos, bem como os possíveis benefícios e os prejuízos das atividades domésticas na gestação.

A média de escores do domínio Atividade física apresentou o menor percentual em relação à nota máxima possível (40\%) dentre todos os domínios investigados. Esses dados demonstram uma necessidade emergente de atender ao recente parecer do American College of Obstetricians and Gynecologists ${ }^{(26)}$, que considera que a atividade física deva ser encorajada à todas as gestantes de baixo risco e aquelas com complicações médicas devem ser avaliadas antes de iniciarem um programa de atividade física.

Esses dados tornam-se reforçados por meio de uma revisão que disseminou informações entre profissionais de saúde que atuam no Brasil sobre as recomendações de atividade física durante a gestação ${ }^{(27)}$. Os autores relataram que mulheres previamente sedentárias devem começar com 15 minutos de exercício aeróbico 3 vezes por semana e aumentar gradativamente 
o tempo de exercícios até o recomendado: 150 minutos de exercício aeróbico por semana, ou 30 minutos de exercício 5 vezes na semana ${ }^{(27)}$.

Diante das diferenças encontradas nas médias e Pmáx de cada domínio estudado, percebe-se a necessidade de uma abordagem específica para a promoção da saúde voltada para temáticas como atividade física, nutrição, sexo seguro e tipo de comportamento no período pré-natal. Referente aos domínios que investigam os riscos associados ao uso de drogas, ao consumo de cigarro e ao de álcool, entretanto, os resultados revelam a conscientização das gestantes possivelmente decorrentes do reflexo de um trabalho efetivo da equipe de saúde.

\section{CONCLUSÃO}

Constatou-se que alguns aspectos do estilo de vida, tais como a atividade física e a nutrição, necessitam de adequação de modo que as gestantes adotem hábitos de vida mais saudáveis por meio de prática de atividade física regular e de alimentação balanceada. Por outro lado, as gestantes parecem conhecer os efeitos negativos associados ao uso de drogas, de álcool e de cigarro.

\section{REFERÊNCIAS}

1. Czneresnia D, Freitas CM. Promoção da saúde: conceitos, reflexões, tendências. Rio de Janeiro: Fiocruz; 2012.

2. Ministério da Saúde (BR). Protocolos da atenção básica: saúde das mulheres. Brasília: Ministério da Saúde; 2016.

3. Dytz JLG, Rocha SMM. O modo de vida da mãe e a saúde infantil. Rev Bras Enferm. 2002;55(2):151-6.

4. Jobim R, Aerts D. Mortalidade infantil evitável e fatores associados em Porto Alegre, Rio Grande do Sul, Brasil, 20002003. Cad Saúde Pública. 2008;24(1):179-87.

5. World Health Organization - WHO. Health promotion glossary. Geneva: WHO; 1998.

6. Cres MR, Abdala GA, Meira MDD, Teixeira CA, Ninahuaman MFML, Moraes MCL. Religiosidade e estilo de vida de uma população adulta. Rev Bras Promoç Saúde. 2015;28(2):240-50.

7. Dietz WH, Douglas CE, Brownson RC. Chronic disease prevention: tobacco avoidance, physical activity, and nutrition for a healthy start. JAMA. 2016;316(16):1645-6.

8. Pôrto EF, Kümpel C, Castro AAM, Oliveira IM, Alfieri FM. Como o estilo de vida tem sido avaliado: revisão sistemática. Acta Fisiatr. 2015;22(4):199-205.

9. Lin YH, Tsai EM, Chan TF, Chou FH, Lin YL. Health promoting lifestyles and related factors in pregnant women. Chang Gung Med J. 2009;32(6):650-61.

10. Carvalho ML, Almeida CAL, Marques AKL, Lima FF, Amorim LMM, Souza JML. Prevenção da mortalidade materna no pré-natal: uma revisão integrativa. Rev Interd. 2015;8(2):178-84.

11. Wilson DMC, Nielsen E, Ciliska D. Lifestyle assessment: testing the FANTASTIC instrument. Can Fam Physician. 1984;30(1):1863-6.

12. Añez CRR, Reis RS, Pretroski EL. Versão brasileira do questionário "Estilo de vida fantástico": tradução e validação para adultos jovens. Arq Bras Cardiol. 2008;91(2):102-9.

13. Leite TRA, Santos BRM. Pressão arterial e estilo de vida de estudantes universitários. Rev Bras Ciên Saúde. 2011;9(27):1420.

14. Barbosa RR, Martins MCG, Carmo FPT, Jacques TM, Serpa RG, Calil AO, et al. Estudo sobre estilos de vida e níveis de estresse em estudantes de medicina. Int J Cardiovasc Sci. 2015;28(4):313-9.

15. Tavares JS, Melo ASO, Amorim MMR, Barros VO, Takito MY, Benício MHD, et al. Padrão de atividade física entre gestantes atendidas pela estratégia saúde da família de Campina Grande - PB. Rev Bras Epidemiol. 2009;12(1):10-9.

16. Silva FT, Costa FS. Avaliação do nível de atividade física durante a gestação. Rev Bras Ginecol Obstet. 2007;29(9):490500 .

17. Francisqueti FV, Souza LMS, Silva REG, Peraçolli JC, Hirakawa HS. Estado nutricional materno na gravidez e sua influência no crescimento fetal. Rev Simbio-Logias. 2012;5(7):1-13.

18. Silva KB, Carvalho CA. Prevalência da lombalgia e sua associação com atividades domésticas em gestantes do município de Itabuna, Bahia. Rev Baiana Saúde Pública. 2011;35(2):387-96. 
19. Freire K, Padilha PC, Saunders C. Fatores associados ao uso de álcool e cigarro na gestação. Rev Bras Ginecol Obstet. 2009;31(7):335-41.

20. Calheiros CAP, Grijó DO, Rodrigues EOMA, Carvalho FNA, Vilela SC, Leite EPRC, et al. Fatores que interferem na qualidade do sono da gestante no segundo e terceiro trimestre gestacional. Rev Enferm UFPE Online. 2013;7(12):6808-13.

21. Schetter CD, Tanner L. Anxiety, depression and stress in pregnancy: implication for mothers, children, research, and practice. Curr Opin Psychiatry. 2012;25(2):141-8.

22. Ministério da Saúde (BR). Protocolo Clínico e Diretrizes Terapêuticas (PCDT): Atenção integral à pessoas com infecções sexualmente transmissíveis (IST). Brasília: Ministério da Saúde; 2015.

23. Silva R, Jansen K, Souza L, Quevedo L, Barbosa L, Moraes I, et al. Sociodemographic risk factors of perinatal depression: a cohort study in the public health care system. Rev Bras Psiquiatr. 2012;34(2):143-8.

24. Faisal-Cury A, Menezes PR. Antenatal depression strongly predicts postnatal depression in primary health care. Rev Bras Psiquiatr. 2012;34(4):446-50.

25. Thiengo DL, Pereira PK, Santos JFC, Cavalcanti MT, Lovisi GM. Depressão durante a gestação e os desfechos na saúde do recém-nascido: coorte de mães atendidas em unidade básica de saúde. J Bras Psiquiatr. 2012;61(4):214-20.

26. The American College of Obstetricians and Gynecologists. Physical activity and exercise during pregnancy and the post partum period. Committee Opinion. 2015; 650:1-8.

27. Nascimento SL, Godoy AC, Surita FG, Silva JLP. Recomendações para a prática de exercício físico na gravidez: uma revisão crítica da literatura. Rev Bras Ginecol Obstet. 2014;36(9):423-31.

28. Wisniewski D, Gróss G, Bittencourt R. A influência da sobrecarga de trabalho do enfermeiro na qualidade da assistência pré-natal. Rev Bras Promoç Saúde. 2014;27(2):177-82.

\section{Endereço para correspondência:}

Luilma Albuquerque Gurgel

Universidade Estadual do Ceará - UECE

Programa de Pós-Graduação em Saúde Coletiva

Av. Dr. Silas Munguba, 1700.

Bairro: Campus do Itaperi

CEP: 60.714-903 - Fortaleza - CE - Brasil

E-mail: luilma.gurgel@uece.br 